\title{
Design considerations for the use of laser-plasma accelerators for advanced space radiation studies
}

\author{
T. K Ö N I GSTEIN ${ }^{1}$, O. KA R GER ${ }^{1}$, G. PRETZLER ${ }^{1}$, J. B. R OSE N ZW E I G ${ }^{2}$ \\ and B. H I D I N G ${ }^{1,2}$ \\ ${ }^{1}$ Institute of Laser and Plasma Physics, Heinrich-Heine-University Düsseldorf, 40225 Düsseldorf, Germany \\ (hidding@ucla.edu) \\ ${ }^{2}$ Department of Physics and Astronomy, University of California, Los Angeles, CA 90095, USA
}

(Received 30 September 2011; revised 13 January 2012; accepted 18 January 2012; first published online 16 February 2012)

\begin{abstract}
We present design considerations for the use of laser-plasma accelerators for mimicking space radiation and testing space-grade electronics. This novel application takes advantage of the inherent ability of laser-plasma accelerators to produce particle beams with exponential energy distribution, which is a characteristic shared with the hazardous relativistic electron flux present in the radiation belts of planets such as Earth, Saturn and Jupiter. Fundamental issues regarding laser-plasma interaction parameters, beam propagation, flux development, and experimental setup are discussed.
\end{abstract}

\section{Introduction}

At the end of the 20th century, laser systems and laserplasma interaction experiments were already capable of producing relativistic electron beams with multi-MeV energies and even up to hundreds of $\mathrm{MeV}$ with exponential energy distribution (Malka et al. 2002). Since the first demonstration of the production of quasimonoenergetic beams with laser-plasma accelerators (LPAs) in 2004 (Faure et al. 2004; Geddes et al. 2004; Mangles et al. 2004), the main goal of the laser-plasma acceleration community was to increase the energy and to decrease the energy spread, divergence, and emittance of electron bunches. These goals have been met with great success, and contributed to a booming research field. At the same time, laser-plasma technology and acceleration techniques have advanced substantially, and LPA facilities are now mushrooming all over the world.

In addition to this trend, here we present design considerations on a novel application of LPAs with potentially large scientific and economic impact, which makes use of the very same exponential-energy electron beams which have been already available from LPAs for more than a decade. Generating exponential particle beams is the inherent domain of LPAs. In contrast, typical classical particle beam sources, such as linacs and cyclotrons, produce particle beams with very narrow energy spread. This is the result of the completely different injection and acceleration processes involved. For example, in a linac the cavities accelerate injected electrons in phase and naturally lead to monoenergetic beams. In contrast, most LPA scenarios lead to exponential-energy beams. For example, when making use of underdense, gaseous target media, the laser pulse travels with a group velocity $v_{\mathrm{g}}<c$ in the plasma, and therefore the accelerating trailing plasma blowout cavity travels with a phase velocity, $v_{\mathrm{ph}}=v_{\mathrm{g}}<c$, too. Therefore, electrons that are accelerated to relativistic energies and travel at velocities $\sim c$ are faster than the accelerating cavity, which means that they will dephase and move forward within the plasma blowout cavity. Within the blowout, the accelerating field is in dependence to the longitudinal position, the electric field at the bubble end being the highest, and being zero in the middle of the bubble. Since in addition the dimensions of the plasma bubble are typically of the order of tens of microns, only the accelerating electric field and thus the energy, which individual electrons obtain, change in the order of $\mathrm{GV} / \mathrm{m}$ depending on their initial time of injection and momentum. A typical result of such an acceleration scenario is a beam with exponential energy distribution. When instead laser-plasma interaction with overdense, solid media is used, this also typically leads to exponentialenergy electron beams. In that case the laser pulse cannot propagate through the medium but is reflected. However, some $10 \%$ of the laser energy is converted irreversibly into kinetic energy of electrons due to the figure-of8 movement of electrons in the oscillating, focused, transient, and reflected electromagnetic laser pulse field. This results in an electron beam with exponential energy distribution propagating in the forward direction with a broad divergence angle of the order of some $20^{\circ}$. The difference of the exponential-energy electron beams from laser-underdense and laser-overdense interaction is that with laser-gaseous interaction, typically the electron energies obtained are much higher and the divergence angle is much smaller than with laser-solid interaction.

Such exponential-energy beams have so far mostly been regarded as undesired on the way toward monoenergetic electron beams. However, it has recently been recognized that very similar exponential-energy particle 
(a)

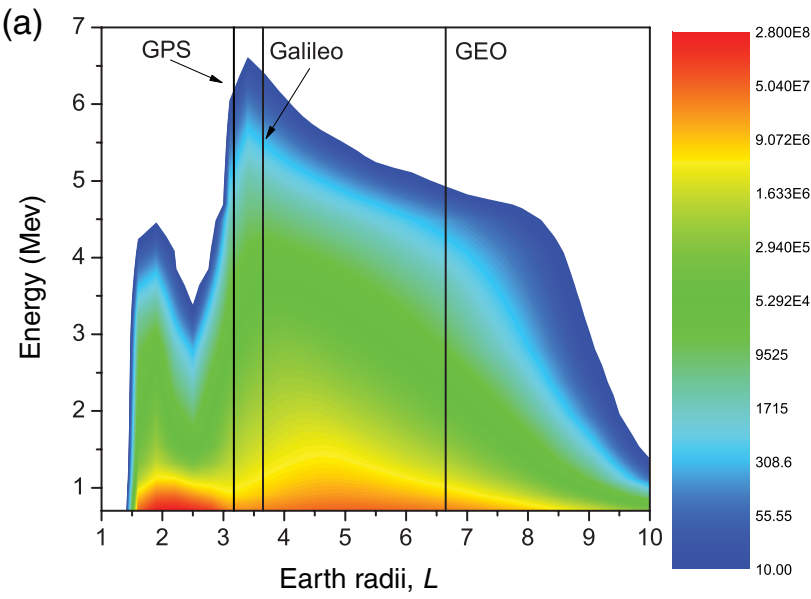

(b)

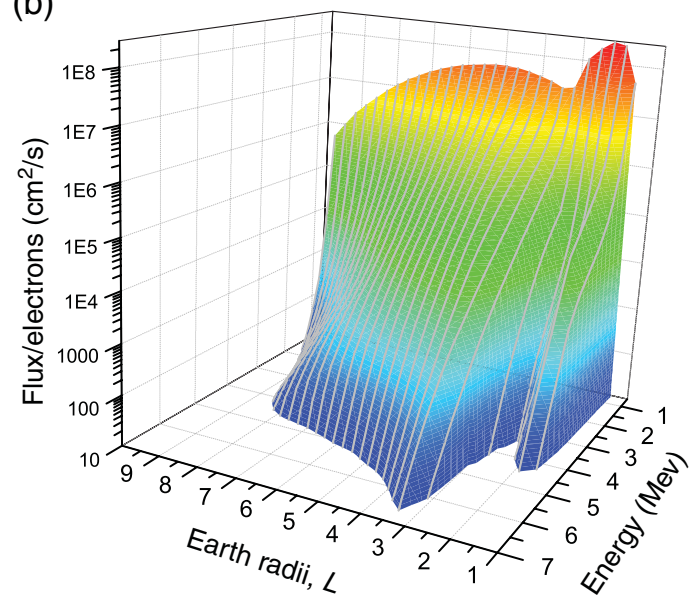

Figure 1. (Colour online) Van Allen belt spectral electron flux according to NASA's AE $8_{\max }$ model (NASA 2011) at distances from Earth of $L=1-10$. In a 2D visualization in (a), the orbits of GPS and Galileo satellites, as well as the geostationary (GEO) orbit, are indicated, while the $3 \mathrm{D}$ visualization $(\mathrm{b})$ indicates the exponential distribution of the spectral flux.

flux exists in space too, and that LPAs can be highly efficient and advanced tools to reproduce such a radiation here on Earth (Hidding et al. 2010, 2011). For example, in the radiation belts of planets with magnetic fields, such as Earth, Saturn and Jupiter, energetic electrons are accelerated and form intense, directed electron flux with exponential energy distribution. These energetic electrons are a substantial threat for satellites and other spacecraft, since they can severely damage electronics on board and solar cells for power generation, for example. This has to be seen in the context of the fact that radiation hardness quality testing, management, and assurance are among the most resource-draining processes in spacecraft design and technology (Garrett 1998), but is also one of the most important issues of space exploration since it can decide if a mission fails or succeeds in the harsh radiation environment of space.

\section{Radiation belt electrons}

The radiation belts of Earth, the van Allen belts, consist of an inner and an outer radiation belt, separated by a 'safe zone'. While the inner radiation belt is dominated by energetic protons, the outer radiation belt is dominated by electrons with energies up to $\sim 10 \mathrm{MeV}$, sometimes dubbed as 'killer electrons' due to their hazardousness for electronics as well as biological systems. The outer radiation belt typically extends from an altitude of about 3 to 10 Earth radii $R_{E}$, corresponding to some 13,000 to $60,000 \mathrm{~km}$, with the highest electron flux usually encountered between $4-5 R_{E}$. Understanding of the nature of the acceleration mechanisms, which do involve plasma waves, is subject to ongoing research (Horne et al. 2005; Chen et al. 2007; Horne 2007). Same holds for the mapping and prediction of particle flux in the radiation belts, which is also a highly active field of research since the discovery of the van Allen belts (Van Allen and Frank 1959). The electron flux within the belts, as well as the extension of the belts, can vary substantially and is connected to solar activity. Figure 1(a) gives an overview on the electron flux predicted by NASA's standard model AE $8_{\max }$ (NASA 2011) during solar maximum activity for Earth radii ranging from $L=1-10$ in the energy range of $E=0.1-7 \mathrm{MeV}$. As shown in Figure 2, during minimum solar activity, the values for the electron flux are lower by a factor of 3 , while the spectral shape stays the same. As can be seen, the electron flux is especially strong at Earth distances, which are used by satellite systems near medium Earth orbit (MEO) as important as GPS and upcoming Galileo, and where the geosynchronous orbit (GEO) is located. The same data are visualized in a $3 \mathrm{D}$ color plot in Fig. 1(b). The z-axis is scaled logarithmically, and the grid lines indicate clearly that the flux energy distribution mostly follows an exponential decay for different $L$-values.

This means that the energy distribution of the van Allen belt electron flux for certain $L$-positions can be described roughly via $N(E)=N_{0} e^{-E / k_{B} T}-$ a feature well known from electron beams generated by LPAs. In LPA research, $k_{B} T=T_{\text {eff }}$ is called the effective temperature of the beam. This indicates that the directed electron flux on stationary orbits can be reproduced by exponential electron beams from LPAs by varying the temperature of the LPA-generated electron beam. This would constitute a fundamental advance, since the current state-of-the-art in radiation testing does so far not involve reproducing the exponential electron flux - simply because particle sources such as LPAs, which enable controlled, tunable exponential-energy flux, have hitherto not been known in the radiation testing community - but instead uses more simplified methods, making use of more conventional particle sources. For example, one basic and widely used approach is to use radiation generated by radioactive sources such as ${ }^{60} \mathrm{Co}(\gamma$-rays and electrons) to evaluate total-dose effects. By varying the distance from 

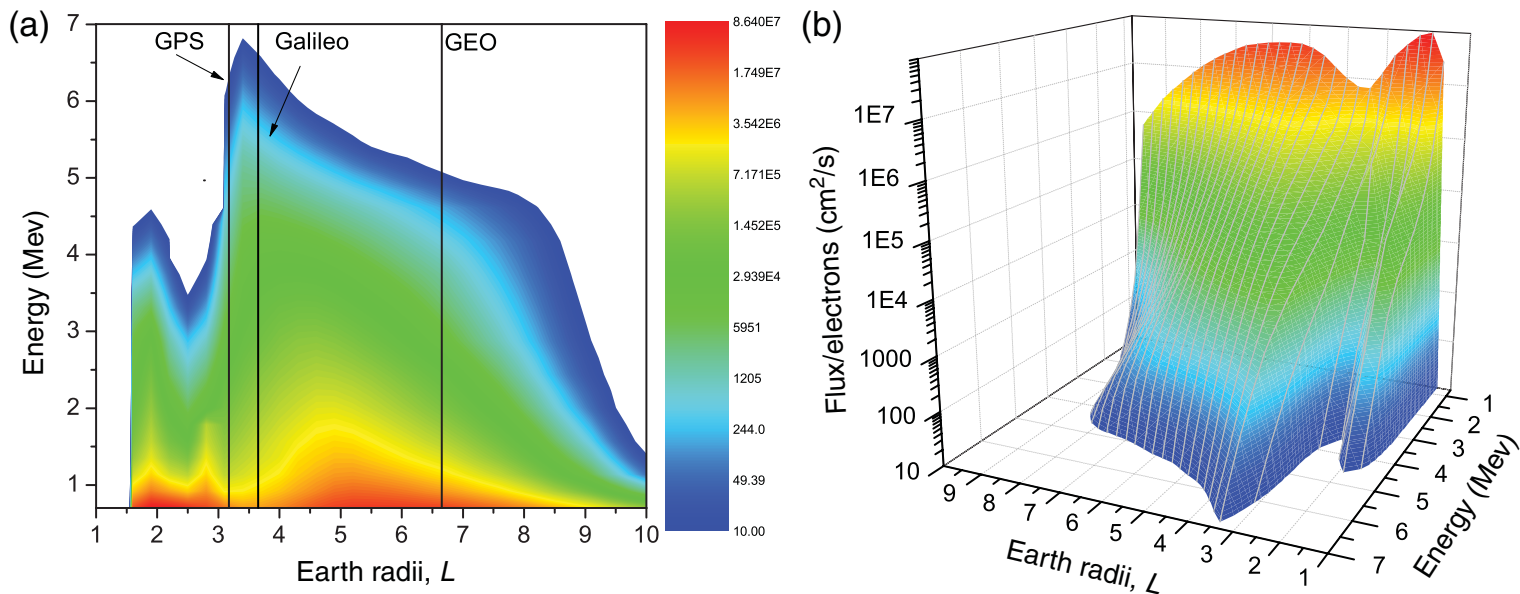

Figure 2. (Colour online) Van Allen belt spectral electron flux according to NASA's AE $8_{\text {min }}$ model (NASA 2011) for solar minima. The spectra are again mostly exponential and only differ from the maximum flux by a factor of 3 .

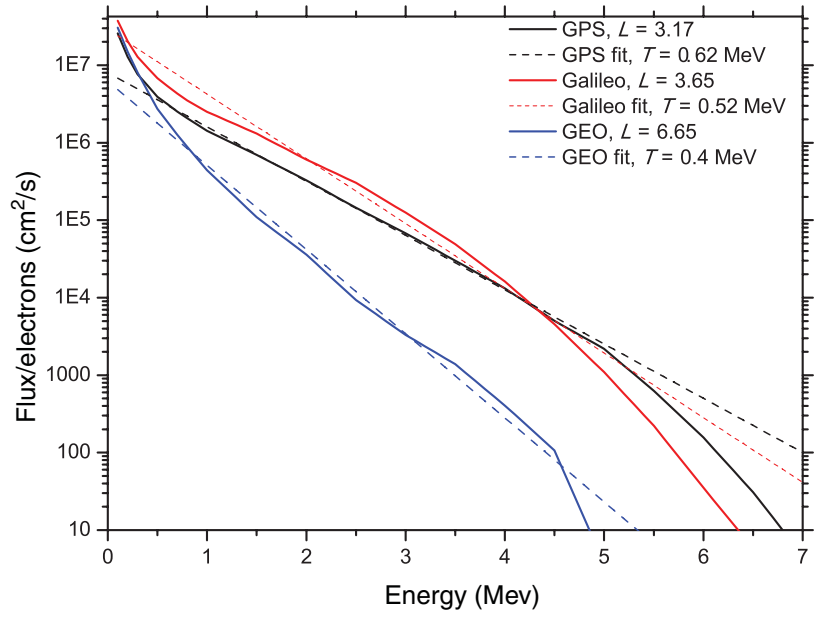

Figure 3. (Colour online) Electron flux according to $\mathrm{AE} 8_{\max }$ at the important orbital distances of GPS, Galileo (both around medium Earth orbits (MEO)) and at geosynchronous orbit (GEO). Fitting exponential particle distributions leads to temperatures in the range of $T_{\text {eff }} \approx 0.4-0.62 \mathrm{MeV}$.

the radioactive source and/or the time of irradiation, the total dose received by the device under test (DUT) is tuned. In addition, to produce electron flux of a certain monoenergetic energy, linacs are widely used. However, such monoenergetic electron flux does not exist in space. For this application, the ability of LPAs to produce flux with exponential energy distribution such as present in the radiation belts is therefore highly desirable.

Figure 3 shows explicitly that at certain fixed orbits the radiation belt electron flux follows an exponential decrease. As an example, the electron flux at $L=3.17$ (GPS), $L=3.65$ (Galileo), and $L=6.65$ (GEO) are plotted. By fitting an exponential decay function, effective temperatures $T_{\text {eff }} \approx 0.4-0.62 \mathrm{MeV}$ can be deduced.

Monoenergetic beams are not well suited to reproduce the specific features of such flux with roughly exponential energy distribution. This is true for flux incident on the space vessel, and also after passing through thin layers of matter - which corresponds to spacecraft shielding and therefore is a very relevant scenario. To illustrate and underline this, Fig. 4(a) shows how a monoenergetic beam of energy $E=4 \mathrm{MeV}$ is decelerated and damped when passing through matter. The flux has been calculated making use of the Monte Carlo code, MULASSIS (Lei et al. 2002), which was developed explicitly for radiation shielding analysis. The marks (i), (ii), (iii), and (iv) correspond to the flux after 1 , 2, 3, and $4 \mathrm{~mm}$ of aluminum. In contrast, Fig. 4 (b) shows how the flux changes when an exponential-energy beam with $T_{\text {eff }}=2 \mathrm{MeV}$ is incident on the same shielding. The spectral flux is damped, but apart from the low-energy cutoff, still has an exponential shape. This behavior is complemented by the results of the total ionizing dose (TID) calculations, which have been carried through for the monoenergetic and exponential-energy beams, and are given in Figs. 4(c) and (d). The TID increases during passage through the solid aluminum in case of the $4 \mathrm{MeV}$ beam (Fig. 4(c)), and reaches its maximum after about $3 \mathrm{~mm}$, when a large fraction of particles has been decelerated down to low energies, and their energy is deposited. In contrast, with the exponential energy flux (Fig. 4(d)) the TID decreases constantly during passage through matter, which is the realistic case expected for radiation belt electrons too. These very fundamental considerations show that exponentialenergy beams are much better suited when compared with monoenergetic beams to reproduce radiation belt electron flux, and thus would enable to develop advanced radiation hardness testing procedures and standards. LPAs are ideally suited as such radiation sources.

Electrons in the outer van Allen belt have maximum energies of up to $E \approx 10 \mathrm{MeV}$. Laser interaction with overdense targets can produce reliably exponential energy electrons in this energy regime with high charge, and is therefore highly suited for this scenario. Furthermore, scaling laws are well known, which predict the electron temperature as a function of laser intensity $I$. These scalings are subject of research since decades and describe the dependence of non-relativistic (Forslund et al. 1977; 

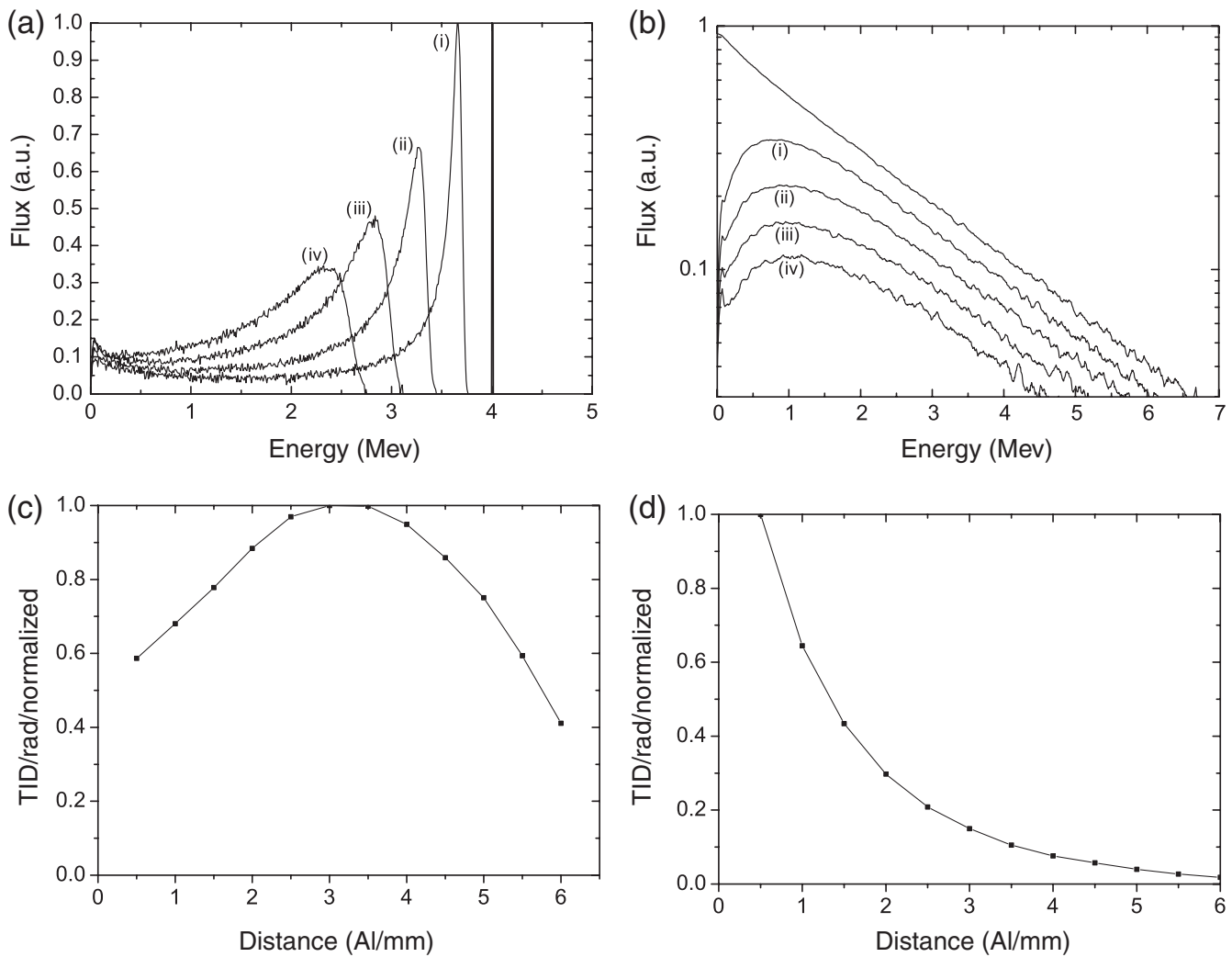

Figure 4. Comparison of spectral flux change of (a) an incident monoenergetic electron beam (as from a linac) of $E=4 \mathrm{MeV}$ when straggling through matter, and (b) an exponential beam as from an LPA with $T_{\text {eff }}=2 \mathrm{MeV}$. The marks (i) to (iv) denote the forward flux after passing through 1 to $4 \mathrm{~mm}$ of aluminum. In contrast to the monoenergetic beam, the exponential-energy beam remains exponential with the exception of the low-energy cutoff. (c) and (d) show the total ionizing dose (TID), which is deposited in a 6-mm tick aluminium wall after irradiation by monoenergetic and exponential beams, respectively.

Estabrook and Kruer 1978; Harrach and Kidder 1981) as well as relativistic laser intensities (Gibbon and Förster 1996). Empirically, a power-law scaling $T_{\mathrm{eff}} \propto\left(I \lambda^{2}\right)^{\zeta}$, where $\lambda$ is the laser wavelength, describes the effective electron temperature, where $\zeta$ typically ranges between $1 / 2$ and $1 / 3$ (Gibbon and Bell 1992). According to Wilks et al. (1992), $\zeta=1 / 2$ in the intensity range of $I \lambda^{2} \approx$ $1.3 \times 10^{18} \mathrm{~W} \mu \mathrm{m}^{2} / \mathrm{cm}^{2}$ to $I \lambda^{2} \approx 1.4 \times 10^{19} \mathrm{~W} \mu \mathrm{m}^{2} / \mathrm{cm}^{2}$, resulting in the explicit scaling, which can be expressed as $I\left(\mathrm{~W} / \mathrm{cm}^{2}\right)=1.37 \times 10^{18} / \lambda(\mu \mathrm{m})^{2} \times\left(\left(T_{\text {eff }} / m_{0} c^{2}+1\right)^{2}-\right.$ 1) and defines an intensity working point suitable to produce flux with the aim temperature. This scaling has been studied analytically in simulations and experimentally in a wide parameter regime in Pukhov et al. (1999) and Sheng et al. (2000). In another well-known work in the intensity range of some $10^{16} \mathrm{~W} / \mathrm{cm}^{2}$ to $10^{19} \mathrm{~W} / \mathrm{cm}^{2}$ (Beg et al. 1997), a value of $\zeta=1 / 3$ is inferred, leading to a scaling $I\left(\mathrm{~W} / \mathrm{cm}^{2}\right)=T_{\text {eff }}(\mathrm{MeV})^{3} \times 10^{20}$.

Now to produce electron flux with an electron temperature of $T_{\mathrm{eff}} \approx 0.35 \mathrm{MeV}$, according to radiation belt flux at $L=3.5$, the laser intensity on target (assuming that a Ti:Sa laser pulse with a central wavelength of about $\lambda=0.8 \mu \mathrm{m}$ is used) should amount to values between $I_{L=3.5} \approx 3.9 \times 10^{18} \mathrm{~W} / \mathrm{cm}^{2}(\zeta=1 / 2)$ and $I_{L=3.5} \approx 4.3 \times$ $10^{18} \mathrm{~W} / \mathrm{cm}^{2}(\zeta=1 / 3)$. By integrating the differential flux according to NASA AE8, the total fluence on a vessel can be calculated and is the reference for LPA- based testing campaigns. For example, a total number of $\approx 3 \times 10^{12} \mathrm{~cm}^{-2}$ electrons can be estimated to be incident per day on a vessel operating at $L=3.5$ during solar maximum activity. A corresponding practical scenario would be a satellite close to GPS orbits, where the electron flux can increase by about one or two orders of magnitude in rise times of the order of 1-2 days, for example (Varotsou et al. 2008). A typical total charge, $Q \approx 100 \mathrm{nC}$, can be assumed to be emitted in the forward direction per shot by LPA interaction corresponding to $3.5 \%$ of the energy of a 1-J laser pulse, which is a typical energy value for the LPA driver pulse. At an intensity of about $I_{L=3.5} \approx$ $5 \times 10^{18} \mathrm{~W} \mu \mathrm{m}^{2} / \mathrm{cm}^{2}$, producing electron flux with a temperature $T_{\text {eff }} \approx 0.35 \mathrm{MeV}$ one would need only $\approx 5$ laser shots, or half a second of LPA performance at $10 \mathrm{~Hz}$ repetition rate, in order to produce the total flux incident on a vessel at $L=3.5$ in space per day. However, this value is only theoretical and underestimates the total number of shots needed, since one cannot put the DUT directly behind the target, and the electron flux generated via laser-solid interaction is emitted in a cone with broad divergence. The divergence is also intensitydependent (Green et al. 2008; Hidding et al. 2011), and amounts for the above-estimated intensity range to some $25^{\circ}$ full divergence angle. Assuming a radially Gaussian intensity distribution, a DUT with an area of 

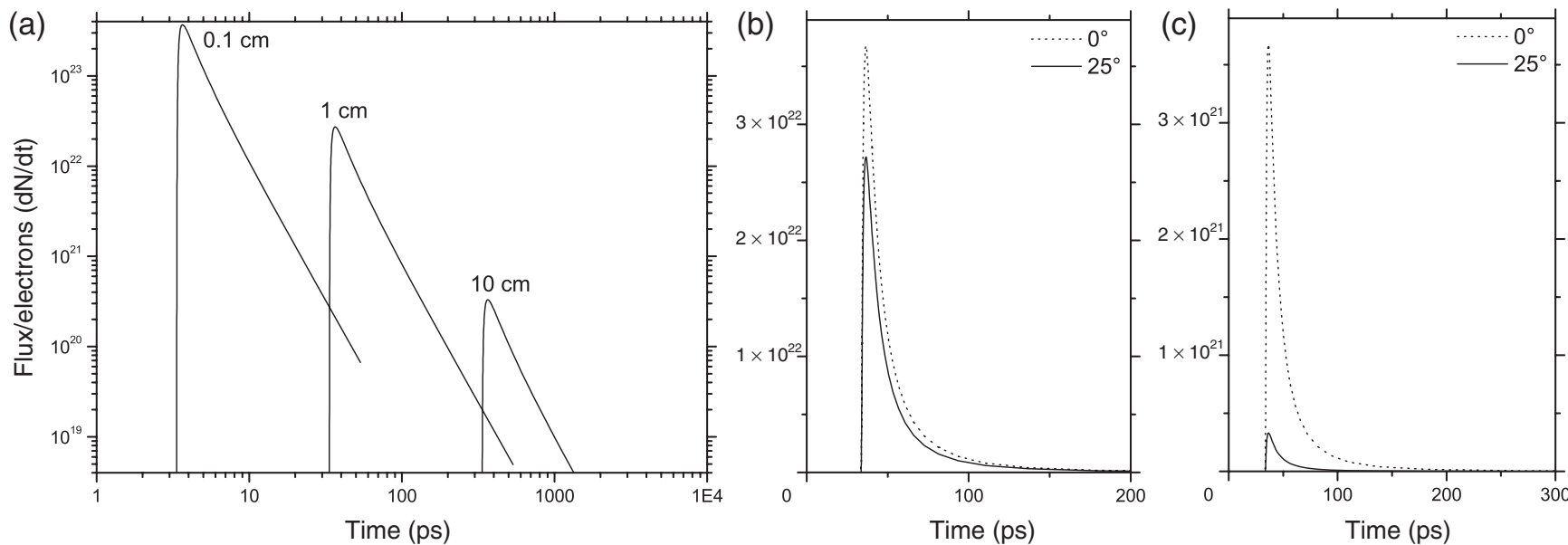

Figure 5. Reduction of exponential-energy electron flux due to energy-dependent velocities and divergence. In (a), the flux of a beam with $T_{\text {eff }}=0.35 \mathrm{MeV}, Q=100 \mathrm{nC}$, and a divergence $\theta=25^{\circ}$ through a DUT area of $1 \mathrm{~cm}^{2}$ is calculated at distances 0.1 , 1 , and $10 \mathrm{~cm}$ behind target (note the logarithmic scaling). Next, the influence of the divergence is visualized by plotting the flux through $1 \mathrm{~cm}^{2}$ after a distance $1 \mathrm{~cm}(\mathrm{~b})$ and $10 \mathrm{~cm}$ (c) for the beam with parameters as in (a), but for a hypothetical divergence of $\theta=0^{\circ}$ and $25^{\circ}$.

$1 \mathrm{~cm}^{2}$, positioned at a distance of $10 \mathrm{~cm}$ away from the radiation source on axis, would need 56 shots in order to receive the maximum daily doses of a satellite surface. Similar to what is done in conventional radiation hardness testing with ${ }^{60} \mathrm{Co}$ sources, the received flux can be tuned by varying the distance. As a further remark, laser systems are currently under development which promise to produce multi- $\mathrm{mJ}$ energies at $\mathrm{kHz}$ repetition rates. Such systems would be focusable to intensities in the $10^{18} \mathrm{~W} / \mathrm{cm}^{2}$ regime. With such systems, the yearly flux on a satellite could be reproduced within seconds in the laboratory. Similarly, it is conceivable to use a highpower, $10-\mathrm{Hz}$ system, and to split the pulse into several delayed laser beams in order to increase the effective repetition rate.

There is a fundamental difference between radiation belt flux and LPA-generated flux. Radiation belt flux is quasi-continuous whereas LPA-generated electron flux is initially pulsed since it is generated during the laser pulse interaction with plasma electrons. At the source the duration of the individual electron beams is equal to the laser pulse duration. However, since we have exponential energy distribution, due to dispersion the time of flight of the electrons to the target is massively different for particles with different energies up to $\approx 1 \mathrm{MeV}$, which leads to a dramatic thinning out and a reduction of peak flux after centimeter scale propagation. Also, the spatial inhomogenity of the beam is reduced in the same way due to the finite opening angle of the beam. As an example, Fig. 5(a) shows the reduction of flux of a LPAgenerated beam with $T_{\text {eff }}=0.35 \mathrm{MeV}, Q=100 \mathrm{nC}$, and a divergence $\theta=25^{\circ}$ through a DUT area of $1 \mathrm{~cm}^{2}$ after distances of $0.1,1$, and $10 \mathrm{~cm}$. This shows that the peak flux is reduced by more than two orders of magnitude, while the beam is stretched out in time. In Fig. 5(b) and (c), the influence of the finite divergence is illustrated by plotting the flux through the $1-\mathrm{cm}^{2}$ test area after
$1 \mathrm{~cm} \mathrm{(b)} \mathrm{and} 10 \mathrm{~cm} \mathrm{(c)} \mathrm{if} \mathrm{zero} \mathrm{divergence} \mathrm{(dashed} \mathrm{line)}$ and $25^{\circ}$ divergence (solid line) are assumed. Here, at a distance of $1 \mathrm{~cm}$ from target, most of the flux still goes through the DUT, while at $10-\mathrm{cm}$ distance, the fraction on axis which will hit the DUT surface is much smaller and the peak flux will be lower.

The pulsed nature of LPA flux in combination with the tunable peak flux due to divergence and exponential energy distribution has positive as well as negative aspects. On the one hand, too high peak flux might lead to 'unnatural' effects, which would not occur in the quasi-continuous, comparably low-flux environment in the radiation belts. On the other hand, the ability to increase flux to high peak levels enables to study the threshold at which such nonlinear effects might occur. This can be also advantageous in the context of both natural as well as unnatural rapid flux release events.

\subsection{Outer planets radiation belt electrons}

Relativistic electrons are present in the radiation belts of all five strongly magnetized planets of our solar system, namely Earth, Jupiter, Saturn, Uranus and Neptune (Mauk and Fox 2010). Mainly because the Jovian magnetic field is the strongest planetary field, and Jupiter's rotation is the fastest of all the planets, electrons reach the highest peak flux and energies in its orbits. Jupiter is currently in the focus of mission planning of both European Space Agency (ESA) as well as NASA, for example because of the possibility of life on Jovian moons. The extreme electron flux environment around Jupiter is a major challenge in the context of these missions. According to current models, which were developed after the first measurements of the Pioneer and Voyager probes (Fillius and McIlwain 1974; Divine and Garrett 1983; Bolton et al. 2002; Garrett et al. 2005; Horne et al. 2008; Tao et al. 2011), the maximum electron energies in Jovian radiation belts can amount 

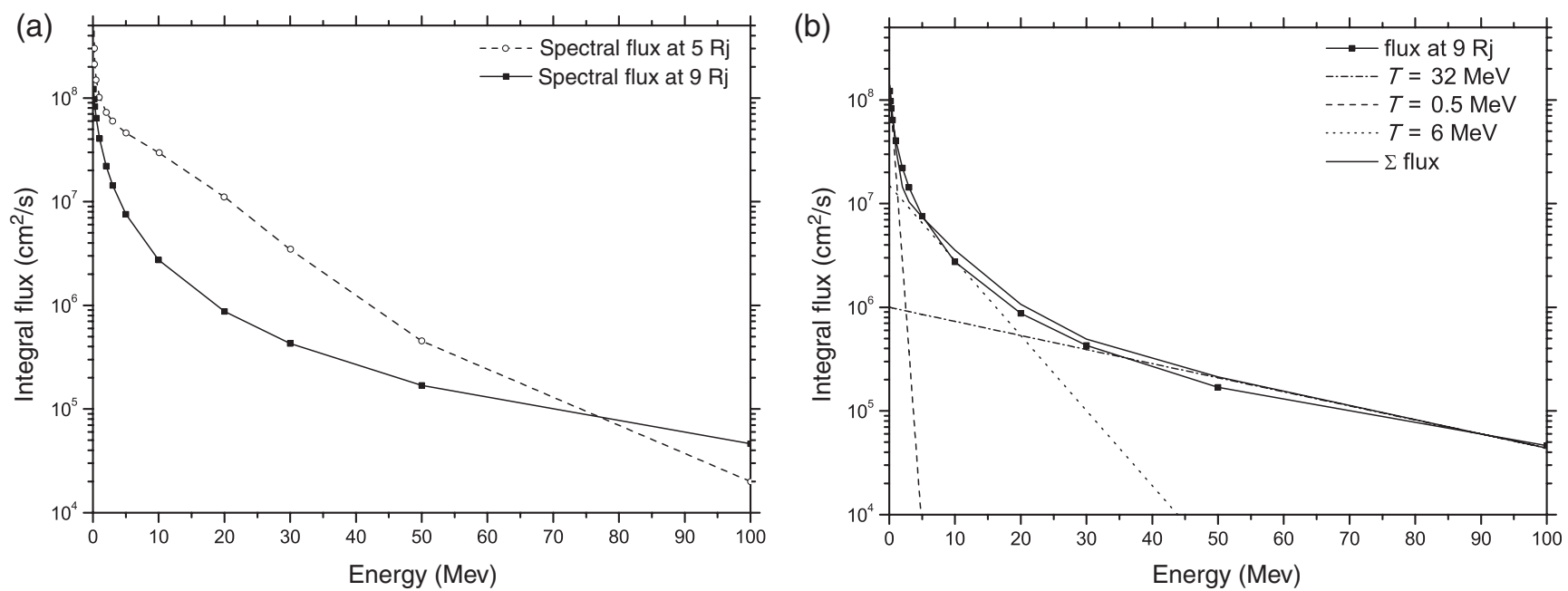

Figure 6. Spectral flux in Jupiter's radiation belt. (a) Flux at a distance of $5 R_{\mathrm{J}}$ and $9 R_{\mathrm{J}}$, (b) overlaying three exponential spectra can reproduce with high accuracy the flux expected at $9 R_{\mathrm{J}}$.

up to $100 \mathrm{MeV}$, maybe more, at flux levels, which can be much higher than in Earth's radiation belts. In Fig. 6(a), as an example, the expected spectral flux is given for two distances around Jupiter, namely at 5 and $9 R_{\mathrm{J}}$. It can be seen that the flux at $5 R_{\mathrm{J}}$ could be approximated by an exponential energy distribution. Although the flux at $9 R_{\mathrm{J}}$ cannot be directly described by a single exponential function, it can be approximated by overlaying various different exponential spectra, as is shown in Fig. 6(b). Here, three different exponential spectra with temperatures of $T_{\text {eff }}=0.5,6$ and $32 \mathrm{MeV}$ are used to approximate the actual expected electron flux.

Such high energies and temperatures suggest the use of laser-underdense interaction to reproduce the Jovian radiation belt electron flux. In addition, it might be useful to additionally shape the resulting electron flux in a plasma wakefield accelerator stage similar to as described in RadiaBeam Technologies (2011) and Rosenzweig et al. (2011). The reproduction and shaping of the extreme electron flux on specific Jovian missions by laser-plasma interaction is a unique possibility to test space electronics which are chosen for these missions in an unprecedentedly realistic environment. This will potentially increase the predictability and confidence level dramatically in mission component reliability, and could reduce the cost for the ESA and NASA missions (approximately with a billion dollar budget) as aimed at for the next decade.

As a side note, the reader from the laser-plasma acceleration community might find it intriguing to follow us with the idea that the natural acceleration processes, which are responsible for electron acceleration at Jupiter (Horne et al. 2008), share a number of similarities with the basic principle of LPAs. Evidently, the mechanics that govern the respective acceleration process in detail are vastly different in an LPA compared with those in a radiation belt, which is clear when considering the vastly different densities, intensities, and time scales of both the processes. The similarities can be found as follows: In the first step volcanic activities on Jupiter's moon Io ejects gaseous matter into the magnetosphere the LPA analogue would be a gas jet nozzle. Next, sunlight and secondary electrons ionize this ejected matter (LPA analogue: the laser pulse). Finally, plasma waves (so-called 'whistler-mode chorus waves') are excited, which, under proper resonance and dephasing conditions ('gyro-resonance', Horne et al. 2008) can accelerate the electrons to relativistic energies. Future activities are currently in preparation which aim at reproducing outer planets' electron environment with LPAs.

\section{Experimental setup for Earth radiation belt reproduction and electronics testing}

We are currently working toward the first demonstration of the potential LPAs for the van Allen belt electron flux mimicking in a ground-based laboratory in an ongoing project. Based on the above considerations, we aim at TNSA-type (Wilks et al. 2001) laser-solid interaction in the intensity range of about $I \approx 5 \times 10^{18} \mathrm{~W} / \mathrm{cm}^{2}$, which is a typical intensity value for the state-of-the-art LPAs and can easily be reached by commercially available systems such as the one described in Willi et al. (2009). In such an interaction scenario, not only electrons but also protons and ions are accelerated during the plasma expansion. Since in this proof-of-principle experiment we aim at using the LPA electron flux only, we will block the expected low-energy protons by a simple Al plate with a thickness of 1 or $2 \mathrm{~mm}$. As a side note, protons and ions within the reach of LPAs are also of interest for space radiation studies (Hidding et al. 2011), but are not considered here since Earth's outer radiation belt are the best suited candidate to demonstrate the potential of LPAs in a proof-of-principle phase.

Figure 7 shows the setup for testing and monitoring of the electron flux. In Fig. 7(a), a compact view is presented, while Fig. 7(b) shows an exploded view of 
(a)

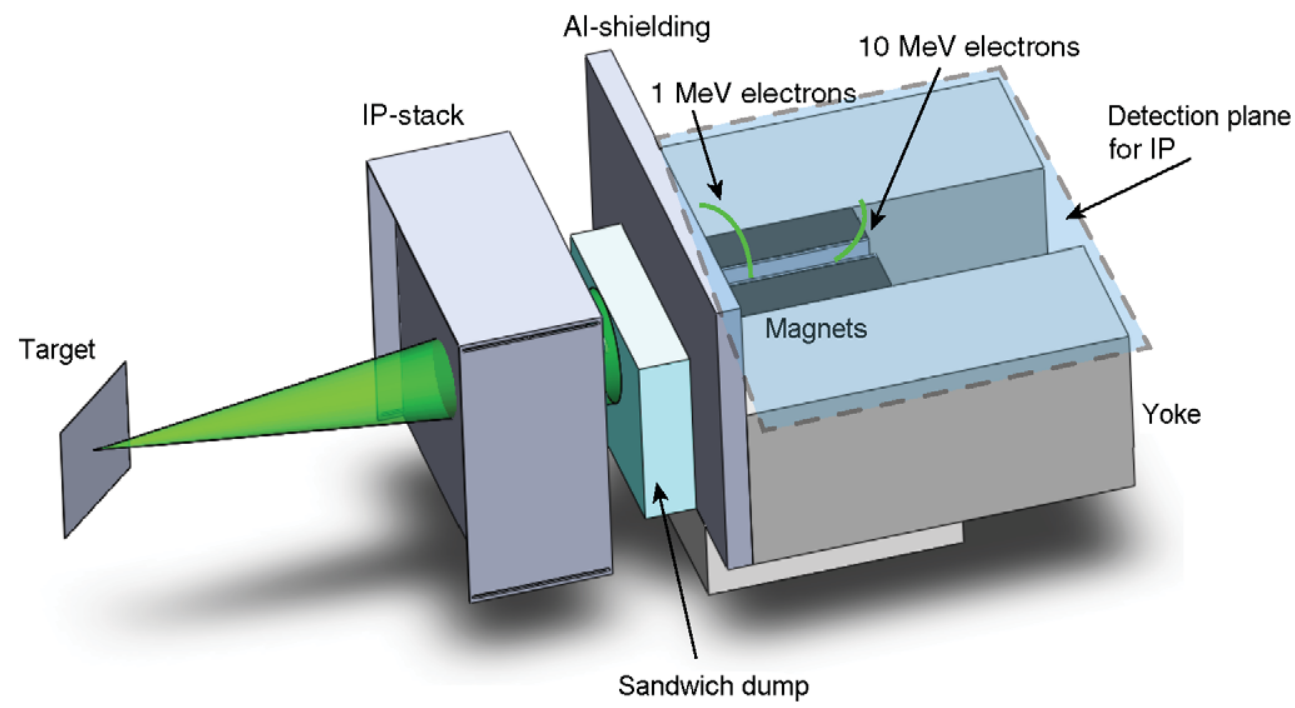

(b)

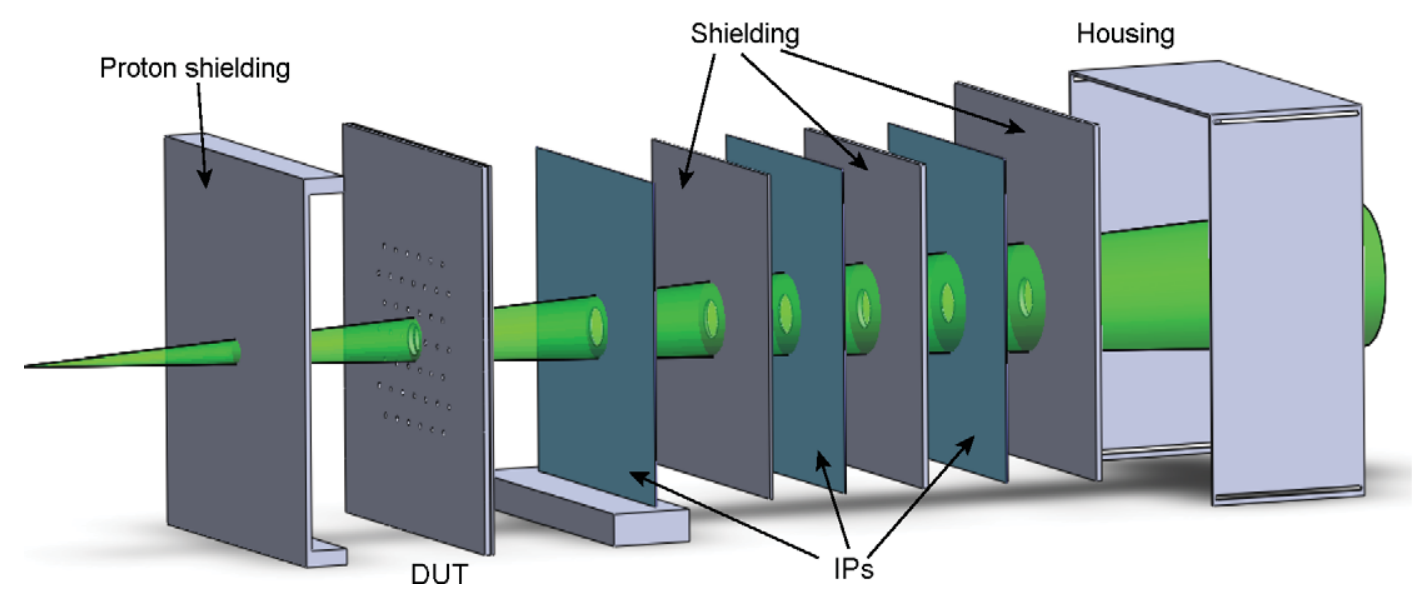

Figure 7. (Colour online) Experimental setup to be used for first irradiation tests. The exponential electron and proton flux from a laser-solid interaction will be incident on an mm-thick shielding for blocking protons and ions. After irradiating the DUT will pass through a sandwich image plate stack for radiation monitoring, and afterwards the electron beam will be further analyzed on axis by monitoring the energy distribution in a magnetic spectrometer (a). In (b) an exploded view of the light and proton shielding plate, the DUT holder plate, as well as the holed IP stack are shown.

the first part of the setup. Behind the proton shield, the DUTs, which in the case of simple components can be devices of the order or $1 \mathrm{~cm}^{2}$ or even smaller, are placed on a holed holding plate. In this case, the DUTs do not block completely the large-divergence electron flux (indicated by the green cone) so that it is possible to monitor the electron flux behind the DUT layer with an electron-sensitive image plate (IP) stack similar as in Hidding et al. (2007) or in Galimberti et al. (2005). Such a stack consists of image plates with a well-defined response function for electrons (Tanaka et al. 2005; Zeil et al. 2010) alternating with beam attenuator layers, for example, simple aluminum plates. The image plates (type: FUJI) are reusable and have a large dynamic range to be used to accumulate many shots without being overexposed so that they can be scanned after irradiation to read out the accumulated electron signal. The Monte-Carlo-type simulations similar to those used to produce Fig. 4 will then be used to reconstruct the incident electron beam flux and temperature. It is especially helpful that the electron sensitivity response of the IPs is nearly constant for electron energies $>1 \mathrm{MeV}$ because the proton shielding does also block $<1 \mathrm{MeV}$ electrons and facilitates the reconstruction of the signal incident on DUTs.

The complete stack as well as the DUT holding plate have a small hole on axis, as is indicated in Fig. 7(b). With an exception of the front proton shielding layer, the incident on-axis fraction of the electron beam can pass the setup without being attenuated, and then enters a dipole magnet spectrometer through an mm-scale aperture to be further analyzed. Electrons are dispersed according to their energy in a permanent magnetic field and are detected by a combination of IP and scintillating screen in the detection plane. The remaining part of the electron beam is dumped in a low-Z-high- $Z$ sandwich 
dump so that these electrons do not falsify the signal as recorded on the energy detection plane IP. This IP can after irradiation be scanned to give further information on the average energy distribution of shots. In addition, a calibrated scintillating screen (Buck et al. 2010) directly behind this IP is used to monitor the energy distribution and intensity and potential shot-to-shot variation online with a triggered CCD camera.

The combination of the described diagnostics enables to have full information on the incident electron flux, and to tune the electron temperature via changing the laser intensity, if necessary. Since the electron flux can be assumed to be radially symmetric, the DUTs should be placed close to the axis on concentric rings with enough space between single DUTs to allow for total flux reconstruction after irradiation by analyzing the IP stack. Finally, it should be mentioned that the temperature slightly off-axis will be slightly lower than onaxis, since the most energetic electrons are emitted in the forward direction on axis. The off-axis temperature dependency can be checked by tilting the diagnostics in the horizontal plane.

\section{Summary}

The requirements on a proof-of-principle experiment aiming to demonstrate for the first time the use of LPAs for ground-based advanced space radiation reproduction and testing of space-grade electronics have been analyzed. Radiation belt electrons mostly have exponential energy distribution, which is the inherent regime of LPA. While exponential-energy electron flux can be produced both via underdense as well as overdense interaction, for the first experiments it makes sense to concentrate on overdense interaction. Maximum radiation belt electron energies and temperatures are well in reach for standard TNSA-type interaction at laser intensities in the range of a few $10^{18} \mathrm{~W} / \mathrm{cm}^{2}$. In such a scenario, few tens of laser shots are sufficient to reproduce for the first time here on Earth with high accuracy the radiation belt fluence, which is incident on a vessel in the outer van Allen belt per day. The spectral flux change and dose deposition when passing through matter such as spacecraft shielding as well as electronic components is substantially different with monoenergetic electron beams from linacs, which are conventionally used, when compared with the LPA-generated beams with matched exponential electron temperature. The use of LPAs therefore promises much more realistic testing for these scenarios, and the development of advanced testing procedures, whereby, for example, for reproduction of the especially harsh Jovian radiation belt electron flux, various beams with varying temperature can be overlayed to approximate the actual spectrum in space. This is similar to advanced treatment plans in particle beam cancer therapy, where different types of particle beams are used to produce the best effect. Due to the initially pulsed nature of LPAgenerated electron beams, extremely high peak fluxes can be produced, which enable campaigns to determine linear radiation effect thresholds and to increase the understanding of radiation effects, whereby the peak flux can be tuned by varying the distance of sample to target due to the exponential energy and connected particle velocity distribution.

\section{Acknowledgements}

This work has been supported by European Space Agency under the NPI programme under contract No. 4000102854 by Strategischer Forschungsfonds, HeinrichHeine-University Düsseldorf, and by the DFG SFB TR18. We would like to thank A. Costantino, E. Daly, V. Ferlet-Cavrois, S. Hernandez, and G. Santin at ESTEC/ ESA; R. D. Harris, P. Adell, H. Schöne, S. McClure, L. Z. Scheick, and J. Insoo at JPL/NASA; A. Murokh, S. Boucher, and P. Frigola at RadiaBeam Technologies; C. Clayton and C. Joshi at UCLA; and E. Esarey, C. G. R. Geddes, W. Leemans at LBNL for fruitful discussions.

\section{References}

Beg, F. N., Bell, A. R., Dangor, A. E., Danson, C. N., Fews, A. P., Glinsky, M. E., Hammel, B. A., Lee, P., Norreys, P. A. and Tatarakis, M. 1997 A study of picosecond laser-solid interactions up to $10^{19} \mathrm{Wcm}^{-2}$. Phys. Plasmas 4(2), 447-457.

Bolton, S. J., Janssen, M., Thorne, R., Levin, S., Klein, M., Gulkis, S., Bastian, T., Sault, R., Elachi, C., Hofstadter, M., et al. 2002 Ultra-relativistic electrons in Jupiter's radiation belts. Nature 415(6875), 987-991.

Buck, A., Zeil, K., Popp, A., Schmid, K., Jochmann, A., Kraft, S. D., Hidding, B., Kudyakov, T., Sears, C. M. S., Veisz, L., et al. 2010 Absolute charge calibration of scintillating screens for relativistic electron detection. Rev. Sci. Instrum. 81(3), 033301.

Chen, Y., Reeves, G. D. and Friedel, R. H. W. 2007 The energization of relativistic electrons in the outer van allen radiation belt. Nat. Phys. 3(9), 614-617.

Divine, N. and Garrett, H. B. 1983 Charged particle distributions in Jupiter's magnetosphere. J. Geophys. Res. 88, 6889-6903.

Estabrook, K. and Kruer, W. L. 1978 Properties of resonantly heated electron distributions. Phys. Rev. Lett. 40(1), 42-45.

Faure, J., Glinec, Y., Pukhov, A., Kiselev, S., Gordienko, S., Lefebvre, E., Rousseau, J. P., Burgy, F. and Malka, V. 2004 A laser-plasma accelerator producing monoenergetic electron beams. Nature 431(7008), 541-544.

Fillius, R. W. and McIlwain, C. E. 1974 Radiation belts of Jupiter. 183(4122), 314-315.

Forslund, D. W., Kindel, J. M. and Lee, K. 1977 Theory of hot-electron spectra at high laser intensity. Phys. Rev. Lett. 39(5), 284-288.

Galimberti, M., Giulietti, A., Giulietti, D. and Gizzi, L. A. 2005 Sheeba: a spatial high energy electron beam analyzer. Rev. Sci. Instrum. 76(5), 053303.

Garrett, H. B. 1998 Space radiation environment. Jet Propulsion Laboratory Technical Report, California Institute of Technology, California, USA. 
Garrett, H. B., Levin, S. M., Bolton, S. J., Evans, R. W. and Bhattacharya, B. 2005 A revised model of Jupiter's inner electron belts: updating the divine radiation model. Geophys. Res. Lett. 32(4), L04104.

Geddes, C. G. R., Toth, C., Tilborg, J. van, Esarey, E., Schroeder, C. B., Bruhwilder, D., Nieter, C., Cary, J. and Leemans, W. P. 2004 High-quality electron beams from a laser wakefield accelerator using plasma-channel guiding. Nature 431(7008), 538-541.

Gibbon, P. and Bell, A. R. 1992 Collisionless absorption in sharp-edged plasmas. Phys. Rev. Lett. 68(10), 1535-1538.

Gibbon, P. and Förster, E. 1996 Short-pulse laser-plasma interactions. Plasma Phys. Control. Fusion 38(6), 769793.

Green, J. S., Ovchinnikov, V. M., Evans, R. G., Akli, K. U., Azechi, H., Beg, F. N., Bellei, C., Freeman, R. R., Habara, H., Heathcote, R., et al. 2008 Effect of laser intensity on fastelectron-beam divergence in solid-density plasmas. Phys. Rev. Lett. 100(1), 015003.

Harrach, R. J. and Kidder, R. E. 1981 Simple model of energy deposition by suprathermal electrons in laser-irradiated targets. Phys. Rev. A 23(2), 887-896.

Hidding, B., Königstein, T., Willi, O. and Pretzler, G. 2010 Method for testing the radiation hardness of electronic devices with particle and photon beams generated by laserplasma-interaction. German Patent AZ 102010 010 716.6.

Hidding, B., Königstein, T., Willi, O., Rosenzweig, J. B., Nakajima, K. and Pretzler, G. 2011 Laser-plasmaaccelerators - a novel, versatile tool for space radiation studies. Nucl. Instrum. Methods Phys. Res. A 636(1), 3140.

Hidding, B., Pretzler, G., Clever, M., Brandl, F., Zamponi, F., Lübcke, A., Kämpfer, T., Uschmann, I., Förster, E., Schramm, U., et al. 2007 Novel method for characterizing relativistic electron beams in a harsh laser-plasma environment. Rev. Sci. Instrum. 78(8), 083301.

Horne, R. B. 2007 Plasma astrophysics: acceleration of killer electrons. Nat. Phys. 3(9), 590-591.

Horne, R. B., Thorne, R. M., Glauert, S. A., Douglas Menietti, J., Shprits, Y. Y. and Gurnett, D. A. 2008 Gyro-resonant electron acceleration at jupiter. Nat. Phys. 4(4), 301-304.

Horne, R. B., Thorne, R. M., Shprits, Y. Y., Meredith, N. P., Glauert, S. A., Smith, A. J., Kanekal, S. G., Baker, D. N., Engebretson, M. J., Posch, J. L., et al. 2005 Wave acceleration of electrons in the van allen radiation belts. Nature 437(7056), 227-230.

Lei, F., Truscott, R. R., Dyer, C. S., Quaghebeur, B., Henderickx, D., Nieminen, R., Evans, H. and Daly, E. 2002 Mulassis: a geant4-based multi-layered shielding simulation tool. IEEE Trans. Nucl. Sci. 49(6), 2788-2793.

Malka, V., Fritzler, S., Lefebvre, E., Aleonard, M.-M., Burgy, F., Chambaret, J.-P., Chemin, J.-F., Krushelnick, K., Malka, G., Mangles, S. P. D., Najmudin, Z., Pittman, M., Rousseau, J.-P., Scheurer, J.-N., Walton B. and Dango, A. E. 2002 Electron acceleration by a wake field forced by an intense ultrashort laser pulse. Science 298(5598), 1596-1600.

Mangles, S. P. D., Murphy, C. D., Najmudin, Z., Thomas, A. G. R., Collier, J. L., Dangor, A. E., Divall, E. J., Foster, P. S., Gallacher, J. G., Hooker, C. J., et al. 2004 Monoenergetic beams of relativistic electrons from intense laser-plasma interactions. Nature 431(7008), 535-538.

Mauk, B. H. and Fox, N. J. 2010 Electron radiation belts of the solar system. J. Geophys. Res. 115, A12220.

NASA 2011 Ae-8 radiation belt models at spdf. accessed September 1, 2011. http://modelweb.gsfc.nasa.gov/models/ trap.html.

Pukhov, A., Sheng, Z.-M. and ter Vehn, J. M. 1999 Particle acceleration in relativistic laser channels. Phys. Plasmas 6(7), 2847-2854.

RadiaBeam Technologies. 2011 Method for testing electronic components. Extended United States patent serial no. 13/042,738. RadiaBeam Technologies, Santa Monica, California.

Rosenzweig, J. B., Valloni, A., Alesini, D., Andonian, G., Bernard, N., Faillace, L., Ficcadenti, L., Fukusawa, A., Hidding, B., Migliorati, M., et al. 2011 Design and applications of an x-band hybrid photoinjector. Nucl. Instrum. Methods Phys. Res. A 657(1), 107-113.

Sheng, Z. M., Sentoku, Y., Mima, K., Zhang, J., Yu, W. and Meyer-ter Vehn, J. 2000 Angular distributions of fast electrons, ions, and bremsstrahlung $\mathrm{x} / \gamma$-rays in intense laser interaction with solid targets. Phys. Rev. Lett. 85, 53405343.

Tanaka, K. A., Yabuuchi, T., Sato, T., Kodama, R., Kitagawa, Y., Takahashi, T., Ikeda, T., Honda, Y. and Okuda, S. 2005 Calibration of imaging plate for high energy electron spectrometer. Rev. Sci. Instrum. 76(1), 013507.

Tao, X., Thorne, R. M., Horne, R. B., Ni, B., Menietti, J. D., Shprits, Y. Y. and Gurnett, D. A. 2011 Importance of plasma injection events for energization of relativistic electrons in the Jovian magnetosphere. J Geophys. Res. A 116(1).

Van Allen, J. A. and Frank, L. A. 1959 Radiation around the earth to a radial distance of $107,400 \mathrm{~km}$. Nature $\mathbf{1 8 3}(4659)$, 430-434.

Varotsou, A., Friedel, R. H., Reeves, G.f D., Lavraud, B., Skoug, R. M., Cayton, T. E. and Bourdarie, S. 2008 Characterization of relativistic electron flux rise times during the recovery phase of geomagnetic storms as measured by the ns41 GPS satellite. J. Atmos. Sol.-Terr. Phys. 70(14), $1745-1759$.

Wilks, S. C., Kruer, W. L., Tabak, M. and Langdon, A. B. 1992 Absorption of ultra-intense laser pulses. Phys. Rev. Lett. 69(9), 1383-1386.

Wilks, S. C., Langdon, A. B., Cowan, T. E., Roth, M., Singh, M., Hatchett, S., Key, M. H., Pennington, D., Mackinnon, A. and Snavely, R. A. 2001 Energetic proton generation in ultra-intense laser-solid interactions. Phys. Plasmas 8(2), $542-549$.

Willi, O., Behmke, M., Gezici, L., Hidding, B., Jung, R., Königstein, T., Pipahl, A., Osterholz, J., Pretzler, G., Pukhov, A., et al. 2009 Particle and $\mathrm{x}$-ray generation by irradiation of gaseous and solid targets with a 100 tw laser pulse. Plasma Phys. Control. Fusion 51(12), 124049.

Zeil, K., Kraft, S. D., Jochmann, A., Kroll, F., Jahr, W., Schramm, U., Karsch, L., Pawelke, J., Hidding, B. and Pretzler, G. 2010 Absolute response of fuji imaging plate detectors to picosecond-electron bunches. Rev. Sci. Instrum. 81(1), 013307. 